\title{
EVALUATION THE ROLE OF BONE MARROW ASPIRATE ON OSTEOGENIC OUTCOME OF MANDIBULAR FRACTURES IN RABBITS
}

\author{
Abdelbadia A. Abdelmabood* and Mona Kamel Tawfic**
}

\begin{abstract}
Purpose: The treatment of fractures involves addressing the biology of fracture repair and the mechanical stability of fracture fixation. Traditionally it has included the addition of bone graft to enhance healing. New advances in the understanding of the cellular and molecular mechanisms of fracture repair have led to the use of growth factors to accelerate bone healing. The aim of this study was to evaluate the effect of bone marrow aspirate on healing activity of rabbit mandibular fractures by histological analysis.
\end{abstract}

Material and Methods :Experimental study was on $\mathbf{3 0}$ male rabbits, classified into Group I, normal control group. Group II, fracture control group formed of 10 rabbits in which traumatic mandibular fractures were done then fixed through wiring without application of bone marrow aspirate. Group III (Bone marrow aspirate) formed of 10 rabbits in which traumatic mandibular fractures were done then fixed through wiring with application of bone marrow aspirate.

Results: Fracture site in bone marrow aspirate group showed peripheral pre-existing bony plates with formation of callus formed mainly by the more regularly arranged osteoid trabeculae rimmed by osteoblastic proliferation being more mature towards the periphery and few immature bony trabeculae

Conclusions : Bone marrow aspirate as source of stem cells seem to be of clinical advantage in acceleration of bone healing.

KEYWORDS: Bone Marrow Aspirate ( BMA ), Mesenchymal Stem Cell (MSC)

\section{INTRODUCTION}

Application of bone marrow aspirate in management of non union or delayed fracture lines considered recent modality in the field of maxillofacial surgery. This due to containing of osteoprogenitors cells and growth factors that contributes to enhancement of healing process. Immediate application of bone marrow concentrate as a recent tehniques has great benefits in regenerative medicine ${ }^{(1,2)}$

* Lecturer of Oral and Maxillofacial Surgery, Faculty of Dentistry, Suez Canal University, Ismalia, Egypt.

** Associate professor of Pharmacology, Faculty of medicine, Suez Canal University, Ismalia, Egypt. 
The enhancement of fracture healing have been demonstrated by a lot of researchers and a lot of methods, materials and modalities in order to enhance the osteogenic effect in fracture healing. Treatment of delayed unions and malunions requires restoration of alignment, stable fixation and in many cases adjunctive measures ${ }^{(3)}$.

Recently, the strategy of immediate stem cell application by transplantation of bone marrow aspirate concentrate (BMAC) has been applied . In the other point of views, the ratio of stem cells in bone marrow aspirates reported by the previous studies as $0.01 \%$ of the nucleated cells ${ }^{(4)}$.

Bone marrow aspirate (BMA) has synergistic effect in the process of bone formation or osteogenesis, this role related to the composition of bone marrow aspirate by the combination of osteoproginator stem cell with endothelial progenitor cells. This combination act directly on outcome of bone formation ${ }^{(5,6)}$.

Osteogenic methods to enhance fracture healing include the use of naturally occurring materials, such as autogenous or allogeneic bone graft or autogenous bone marrow grafts that have been shown to induce or support the formation of bone ${ }^{(3)}$.

Bone marrow transplant has been shown to depend on the presence of stem cells, defined as undifferentiated cells able to divide to produce either more MSC or other forms of fully differentiated cells in form of osteoblasts, chondrocytes, adipocytes, and myoblast. In response to an inducing event, the stem cell may undergo one or more cycle of asymmetric cell division ${ }^{(7)}$.

There is rule in treatments of bony fractures aim to fix the fractured ends together and allow the osteogenic process of ossification and remodeling to occur. Recent studies were concentrated on manipulation of stem cells to demonstrate the effect on healing of malunion fractures. There are a pool of bone forming cells exists outside of bone and are able to be mobilize during the need of ossification process as this the role of endocrine body system ${ }^{(8)}$.

There was study demonstrated how these bone marrow osteoprogenitor cells arrive to the area of bony defect by direct application of bone marrow cells on bony defects in case series of three patients. Although this trail study were aimed to reduce the healing time by direct application of bone marrow cells into a bony matrix, but there was no control in this study ${ }^{(9)}$.

Osteogenesis process of the fracture healing start by immediate formation of haematoma at the fracture sites followed by cytokines release to the area of fracture. Adjacent to the fracture line, there will be differentiation of periosteal bone lining cell into bone formation cells and start of callus formation. The fracture gap filled with immature woven bone that is remodeled over time to more formation of sound lamellar bone $\mathrm{e}^{(\mathbf{1 0}, 11)}$.

Osteoblasts cells as cell of bone formatting cell, has the high majorities of investigations on bone regeneration. As this cell during its development it was developed from mesenchmal stem cells(MSC). These MSC are multipotent cells with the capacity to differentiate into other cell types including chondrocytes,myoctes and CT fibroblasts. MSC found to be persist in bone marrow with ratio $1 / 100,1000$ and found in other locations as liver, spleen and placenta ${ }^{(12)}$.

The aim of this study was to evaluate the effect of bone marrow aspirate on healing activity of mandibular fractures by histological analysis.

\section{MATERIALS AND METHODS:}

30 adult male White New Zealand rabbits with age range 7 to 9 months and body weight about 3.5 to $5 \mathrm{~kg}$ were included in this study. Each rabbit was be housed individually with free access to a standard rabbit pellet diet and water. The rabbits were kept in a dedicated animal house under veterinary 
supervision. The housing room at Laboratory Animal Unit of Faculty of Medicine, Suez Canal University.

Rabbit grouping: 30 male rabbits, classified into

Group I, normal control group formed of 10 rabbits .

Group II, fracture control group formed of 10 rabbits in which traumatic mandibular fractures were done then fixed through wiring without application of bone marrow aspirate.

Group III (Bone marrow aspirate ) formed of 10 rabbits in which traumatic mandibular fractures were done then fixed through wiring with application of bone marrow aspirate.

\section{Surgical procedure:}

General anesthesia was provided using ketamine hydrochloride. The animals were anaesthetized by intramuscular injections of $30 \mathrm{mg} / \mathrm{kg}$ ketamine (Novartis, Basel, Switzerland) and $5 \mathrm{mg} / \mathrm{kg}$ xylazine (Xyla- Ject $20 \mathrm{mg}$ xylazine base $/ \mathrm{ml}$ ). Preoperatively, intramuscular injection of $30 \mathrm{mg} / \mathrm{kg}$ of long acting oxytetracycline (Troy Laboratories Pty Limited, Glendenning, NSW, Australia) and $1.5 \mathrm{mg} / \mathrm{kg}$ of diclofenec sodium ( Novartis, Basel, Switzerland) were injected.

\section{Bone marrow aspirate (BMA) biopsy:}

BMA was done using aspirating needle technique from both tibias of rabbit. The inner surface of a spinal needle and $10 \mathrm{ml}$ syringes were coated with heparin of $1000 \mathrm{U} / \mathrm{ml}$, as the biopsies were sended to laboratory for the following centrifugation steps Bone marrow aspiration was carried out according to Kim et al ${ }^{(13)}$ :

1. With sterile conditions, bone marrow aspirate was diluted at a ratio of 2:1 with calcium magnesium free phosphate-buffered saline .
2. BMA become centrifuged at $2,000 \mathrm{rpm}$ for 20 min at $20 \_C$ in a swinging-bucket rotor without brake.

3. The mononuclear cells were separated from the interface at the top of the Ficoll-Hypaque layer and were with concentrated to be transferred to a new sterile conical falcon tube containing 20 $\mathrm{ml}$ Eagle's medium for centrifugation at 1000 rpm for $10 \mathrm{~min}$.

4. The adherence of cells as well as their growth was carefully monitored, and nonadherent cells were removed from culture after $2 \mathrm{e} 3$ days by a series of medium changes.

5. Adherent cells were expanded as monolayer cultures in a 5\% CO2/95\% air atmosphere at 37 _C with medium changes every $2 \mathrm{e} 3$ days until they reached approximately $70 \% \mathrm{e} 80 \%$.

\section{Mandibular fractures and application of BMA :} Fig (1)

- After skin preparation and disinfection, $1 \mathrm{~cm}$ longitudinal incisions was done below the lower edge of right mandibular body to angle of rabbit followed by blunt dissection of the subcutaneous tissue.

- The platysma was incised and carefully retracted followed by supraperiosteal dissection, elevating the buccinator muscle and exposing of the periosteum were incised and reflected to complete exposure the surgical site.

- Traumatic mandibular fracture were done at the mandibular body of all rabbits with aids of surgical osteotome. Complete mobilization of the two fragments followed by stainless steel wiring as a method of fixation were be done. Application of BMA biopsy for group III to fill over the fracture site followed by complete closure with vicryle $3 / 0$ in layers. 


\section{Postoperative:}

- Postoperative daily injections of $1.5 \mathrm{mg} / \mathrm{kg}$ of diclofenac sodium and $15 \mathrm{mg} / \mathrm{kg}$ of oxytetracycline was administrated for 3 days, and the animals will be observed for any signs of infections or wound dehiscence.

- Four weeks after bone marrow aspirate treatment, rabbits in all groups were sacrificed and tissues were separated and fixed in a $10 \%$ phosphate-buffered paraformaldehyde solution. Tissues were dehydrated, embedded in paraffin and sectioned at $4-\mu \mathrm{m}$ and stained with hematoxylin and eosin (H\&E).

- Then specimens were examined blindly under a light microscope and the degree of fracture repair was graded as: 4- complete bony union; 3 - the bony union was less than complete due to the presence of a small amount of cartilage in the callus; 2 complete cartilaginous union; 1retention of fibrous elements in the cartilaginous plate; 0-formation of a pseudo arthrosis (Allen et al, 1980) $)^{(14)}$.

Results were collected and expressed as Mean \pm SD. Results were analyzed using the statistical package for the social sciences, version 20 (SPSS Software, SPSS Inc., Chicago, USA). One-way analysis of variance (ANOVA) followed by Duncan's post-hoc test were used to test the significance of the difference between quantitative variables. $\mathrm{P}$ value $<$ 0.05 was considered to be statistically significant.

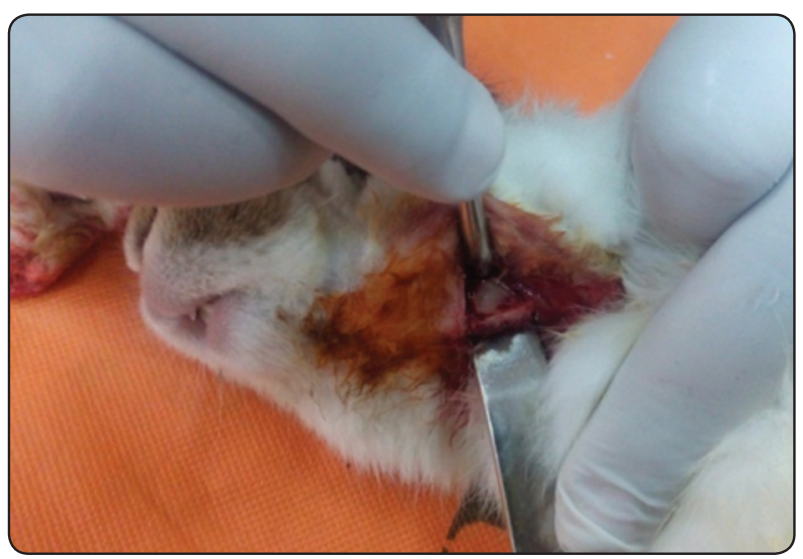

Fig. (1) Mandibular fracture with blunt dissection

\section{RESULTS}

The aim of this study was to evaluate the effect of bone marrow aspirate on healing activity of mandibular fractures by histological analysis. 30 adult male White New Zealand rabbits classified into Group I, normal control group formed of 10 rabbits . Group II, fracture control group formed of 10 rabbits in which traumatic mandibular fractures were done then fixed through wiring without application of bone marrow aspirate. Group III ( Bone marrow aspirate ) formed of 10 rabbits in which traumatic mandibular fractures were done then fixed through wiring with application of bone marrow aspirate.

The histopathological picture of the fracture specimens from the experimental groups stained with hematoxylin \& eosin (H\&E) are demonstrated as in section (A) Fig (2) in which the normal control groups. Section B_FIG (3) for in fracture site showed peripheral pre-existing bone at edges of fracture and central loose fibrous tissue infiltrated by few inflammatory cells and many proliferating fibroblasts, which show entrapped devitalized bony spicules (deep basophilic ) from the fracture, peripheral areas also showed osteoblastic rimming. And section (C) FIG (4) Fracture site in bone marrow aspirate group showed peripheral preexisting bony plates with formation of callus formed mainly by the more regularly arranged osteoid trabeculae rimmed by osteoblastic proliferation being more mature towards the periphery and few

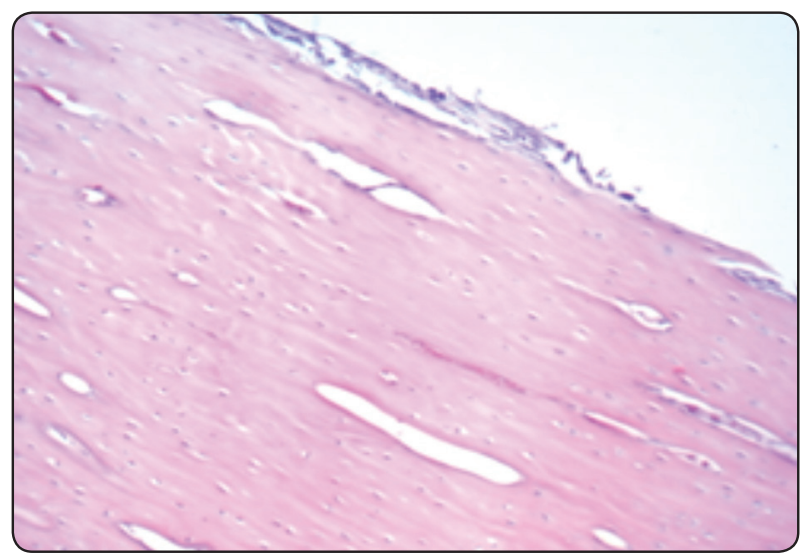

Fig. (2) Histological cross section A 


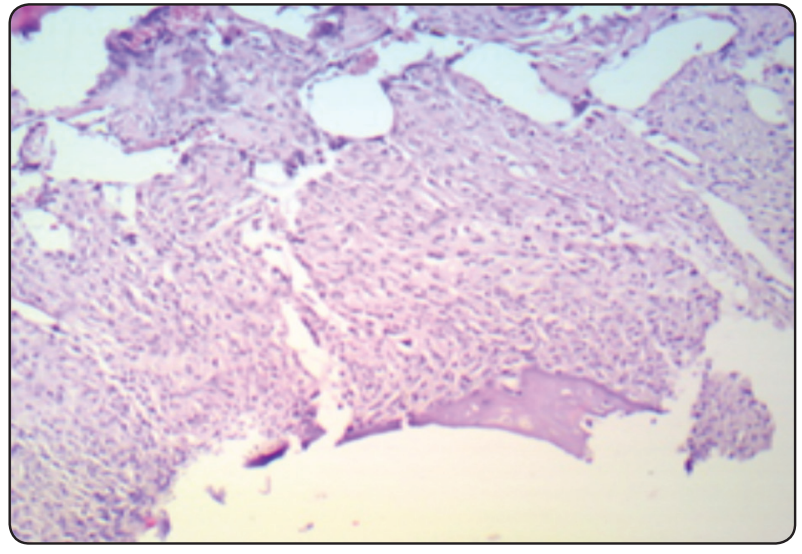

Fig. (3) Histological cross section B

immature trabeculae centrally intervened by spaces in-between trabeculae showed blood elements of early marrow formation, no fibrous elements $(\mathrm{H} \& \mathrm{E} 400 \times)$

The histopathological scores in the experimental groups. Values are expressed as mean \pm S.D. $(n=10)$, analyzed by one-way ANOVA followed by Duncan multiple comparisons test.,"\# $\mathrm{P}<0.05$. Compared with normal control group, ${ }^{\#}$ Compared with fracture control group as in the following graph (1):

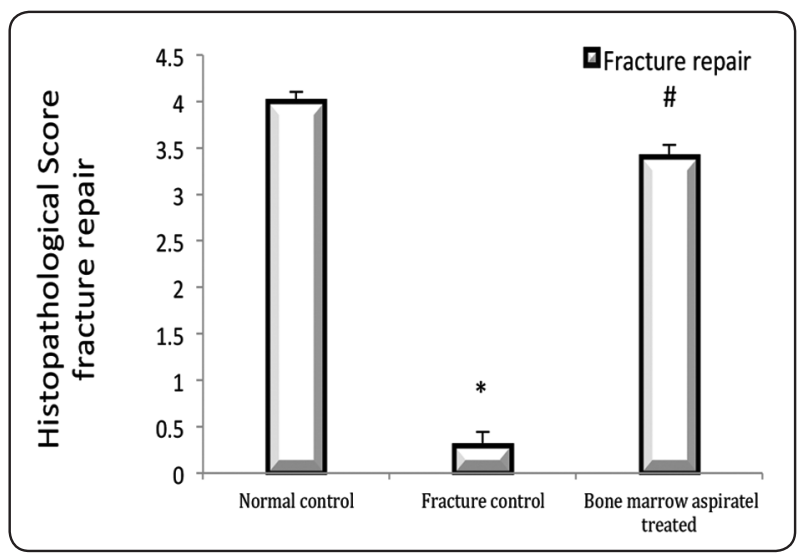

Graph (1): Histopathological scores in the experimental groups

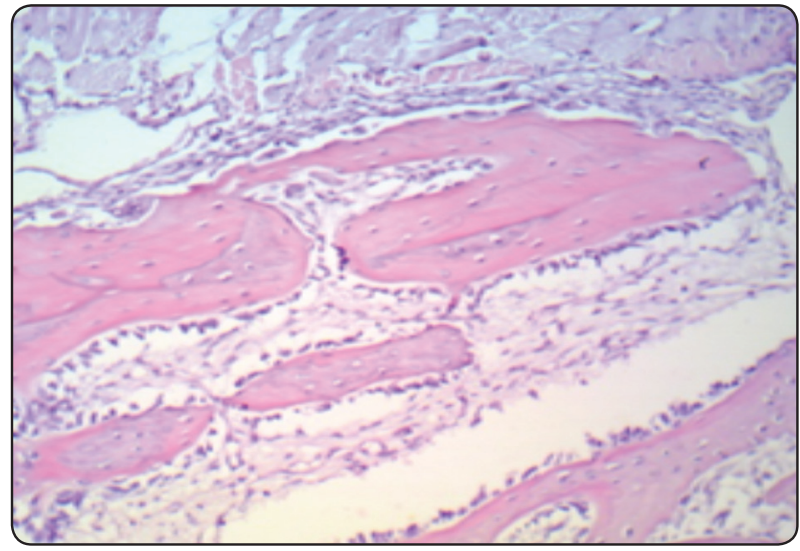

Fig. (4) Histological cross section C

\section{DISCUSSION}

In this study rabbit models were used to study the osteogenic effect of bone marrow aspirate on healing process of mandibular fractures. This was done through application of bone marrow aspirate on fracture lines that were unilaterally for group II and group III .

This fracture lines were created unilaterally at the right region of rabbit mandible. Unlike the bilateral defects that demonstrated in the study by $\boldsymbol{L} \boldsymbol{u}$ and $\boldsymbol{R a p i e}^{(15)}, \mathbf{2 0 0 3}$. This idea of unilateral fracture lines to evaluate the normal control group to differentiate histologically.

This study was concentrated to follow up rabbits till period of 12 weeks as this time was satisfy to investigate the depth of osteogenic effect of bone formation. This was in agreement of Milaro et al ${ }^{(16)}$, 2010 who evaluate the effect of PRP on critical defect in the rabbit models till 3 months. This study by Milaro et $\boldsymbol{a l}^{(16)}, 2010$ suggested that, there will be positive outcome from the effect of PRP appear at 2 week period. On the other hand, the study demonstrated by Bruttil et $\boldsymbol{a l}^{(17)}, 2012$ clarify that, the osteogenic effect on mandibular rabbits should be evaluated exceeding the period of 3 months.

In this study, by examination of bone marrow aspirate rabbit group III (study group) there were 
in the fracture site showed peripheral pre-existing bony plates with formation of callus formed mainly by the more regularly arranged osteoid trabeculae rimmed by osteoblastic proliferation being more mature towards the periphery . This in agreement of the previous studies that demonstrated respectively, as initial vascular invasion of bone healing in rabbits occur on day 5 and full calcifications with complete bone healing occur within 20 day and 40 day ${ }^{(18)}$.

In this study, the histological evaluation of the experimental mandibular defects demonstrated that, there were high capacity for bone formation in the group III ( study group (. From this point of view, we be able to clarify the effect of bone marrow aspirate on healing process of mandibular fracture. This osteogenic effects of bone marrow aspirate, were found in the study group with high rate from beginning as these finding were in agreement of the study by Kon et $\mathbf{a l}^{(19)}, 2000$.

Our study demonstrated, there was regenerated formed bone the group II that show gradual increase in bone volume in histopathological score, but with less than in the study group with high ratio. In histological examination, there were cartilaginous remnants found in group II with increase the fibrous matrix. Where in examination of group III there were increase in size of homogensity of bony arranged osteoid trabeculae.

In this study histopathological score between group II and III as analyzed by one-way ANOVA followed by Duncan multiple comparisons test. P $<0.05$. Compared with normal control group, \# Compared with fracture control group. This increase in regeneration process of mandibular fractures in the study group, may be attributed to the effect of bone marrow aspirate as a source of mesenchymal stem cells and this become in agreement with Jiang et $\boldsymbol{a l}^{(20)} \mathbf{2 0 1 0}$ and Udehiya et $\boldsymbol{a l}^{(21)} \mathbf{2 0 1 3}$, who carified this effect of conversion of MSC to osteoblasts as bony formed cells

\section{CONCLUSIONS}

Bone marrow as a source of mesenchymal stem cell, has the capacity to increase the osteogenic process with benefits in healing process in mandibular fracture in rabbits.

\section{REFERENCES}

1. Chen Y, Shao JZ, Xiang LX, Dong XJ, Zhang GR: Mesenchymal stem cells: a promising candidate in regenerative medicine. Int J Biochem Cell Biol 40(5): 815e820, 2008

2. Farr_e-Guasch E, Prins HJ, Overman JR, Bruggenkate CMT, Schulten EAJM, Helder MN,et al: Human maxillary sinus floor elevation as a model for bone regeneration enabling the application of one-step surgical procedures. Tissue Eng Part B Rev 19(1): 69e82, 2013

3. Susan B, Dobosiu c, Troianescu O, Turcanu B, Some observations on the treatment of pseudarthrosis of the long bones. Chir Narzadow Ruchu Ortop2002; 84-A (3):454-64

4. Murawski C, Kennedy J: Percutaneous internal fixation of proximal fifth metatarsal bones fractures. Am J Sports Med 39(6): $1295 \mathrm{e} 1301,2011$

5. Moioli EK, Clark PA, Chen M, Dennis JE, Erickson HP, Gerson SL, et al: Synergistic actions of hematopoietic and mesenchymal stem/progenitor cells in vascularizing bioengineered tissues. PLoS ONE 3(12): e3922, 2008

6. Atesok K, Li R, Stewart DJ, Schemitsch EH: Endothelial progenitor cells promote fracture healing in a segmental bone defect model. J Orthop Res 28(8): 1007e1014, 2010

7. Fleming JE Jr, Cornell CN, Muschler GF:Bone cells and matrices in orthopedic tissue engineering. Orthop Clin North Am. 2000; 31(3):357-74.

8. Matsumoto T, Kuroda R, Mifune Y, et al. Circulating endothelial/skeletal progenitor cells for bone regeneration and healing. Bone. 2008; 43:434-439.

9. Quarto R, Mastrogiacomo M, Cancedda R, et al. Repair of large bone defects with the use of autologous bone marrow stromal cells. N Engl J Med. 2001; 344:385-386.

10. Lu C, Hansen E, Sapozhnikova A, et al. Effect of age on vascularization during fracture repair. J Orthop Res. 2008; 26:1384-1389.

11. Gruber R, Koch H, Doll BA, et al. Fracture healing in the elderly patient. Exp Gerontol. 2006; 41:1080-1093. 
12. Kassem M, Abdallah BM. Human bone-marrow-derived mesenchymal stem cells: biological characteristics and potential role in therapy of degenerative diseases. Cell Tissue Res. 2008; 331:157-163.

13. Kim H, Lee JH, Suh H: Interaction of mesenchymal stem cells and osteoblasts for in vitro osteogenesis. Yonsei Med J 44(2): 187e197, 2003

14. Allen HL, Wase A, Bear WT. Acta Orthop Scand. Indomethacin and aspirin: effect of nonsteroidal anti-inflammatory agents on the rate of fracture repair in the rat. 1980 Aug; 51(4):595-600.

15. Lu M, Rabie AB: Microarchitecture of rabbit mandibular defects grafted with intramembranous or endochondral bone shown by micro-computed tomography.Br J Oral Maxillofac Surg 41(6): 385e391, 2003.

16. Miloro M, Haralson DJ, Desa V: Bone healing in a rabbit mandibular defect using platelet-rich plasma. J Oral Maxillofac Surg 68(6): 1225e1230, 2010.

17. Busuttil Naudi K, Ayoub A, McMahon J, Di Silvio L, Lappin $\mathrm{D}$, Hunter KD, et al: Mandibular reconstruction in the rabbit using beta-tricalcium phosphate (beta- TCP) scaffolding and recombinant bone morphogenetic protein 7 (rhBMP-7) e histological, radiographic and mechanical evaluations. J Craniomaxillofac Surg 40(8): e461e469, 2012 .

18. Albrektsson T: Repair of bone grafts. Scand J Plast Reconstr Surg 14(1): 1e12, 1980.

19. Kon E, Muraglia A, Corsi A, Bianco P, Marcacci M, Martin I, et al: Autologous bone marrow stromal cells loaded onto porous hydroxyapatite ceramic accelerate bone repair in critical-size defects of sheep long bones. J Biomed Mater Res 5;49(3): 328e337, 2000.

20. Jiang X, Zou S, Ye B, Zhu S, Liu Y, Hu J: bFGF-Modified BMMSCs enhance bone regeneration following distraction osteogenesis in rabbits. Bone 46(4):1156e1161, 2010.

21. Udehiya RK, Amarpal, Aithal HP, Kinjavdekar P, Pawde AM, Singh R, et al: Comparison of autogenic and allogenic bone marrow derived mesenchymal stem cells for repair of segmental bone defects in rabbits. Res Vet Sci 94(3): $743 \mathrm{e} 752,2013$ 